Nouvelles perspectives en sciences sociales

Revue internationale de systémique complexe et d'études relationnelles

\title{
Plurilinguismes, représentations et identités : des pratiques des locuteurs aux définitions des linguistes
}

\section{Cécile Sabatier}

Volume 6, numéro 1, décembre 2010

Sur le thème de la minorité linguistique

URI : https://id.erudit.org/iderudit/1000485ar

DOI : https://doi.org/10.7202/1000485ar

Aller au sommaire du numéro

\section{Éditeur(s)}

Prise de parole

ISSN

1712-8307 (imprimé)

1918-7475 (numérique)

Découvrir la revue

Citer cet article

Sabatier, C. (2010). Plurilinguismes, représentations et identités : des pratiques des locuteurs aux définitions des linguistes. Nouvelles perspectives en sciences sociales, 6(1), 125-161. https://doi.org/10.7202/1000485ar
Résumé de l'article

La présente contribution propose de dégager les discours et les représentations que des adolescents plurilingues tiennent sur leur vécu sociolinguistique, sur le développement de leur plurilinguisme et sur les identités (individuelles, collectives, linguistiques et culturelles) qu'ils développent en contextes. À partir d'entretiens individuels socio-biographiques réalisés auprès de 11 jeunes français-maghrébins, l'auteur s'attache à décrypter les représentations des langues et les stratégies identitaires mobilisées dans des contextes de contacts de langues pour mettre à jour la construction et le développement des plurilinguismes individuels au regard 1) des modalités d'acquisition et de transmission des langues, 2) des répertoires verbaux déclarés et de la pluralité des identités que les sujets revendiquent pour 3) s'interroger sur la manière de caractériser les situations complexes de contact. 


\title{
Plurilinguismes, représentations et identités : des pratiques des locuteurs aux définitions des linguistes
}

\author{
Cécile Sabatier \\ Simon Fraser University, Burnaby
}

l'heure où la société se mondialise, où la mobilité des indi-
vidus est en hausse croissante, et où la diversité linguistique
et culturelle fait l'objet de projets éducatifs explicites ${ }^{1}$, les prati-
ques (socio)langagières et les répertoires verbaux que les individus

1 Cécile Sabatier, Rôle de l'école dans le développement et la construction du plurilinguisme chez des enfants issus de la migration maghrébine en France, Lille, ANRT, 2004; Michel Candelier (dir.), L'éveil aux langues à l'école primaire. Evlang : bilan d'une innovation européenne, Bruxelles, De Boeck, 2003; Réjean Canac-Marquis et Cécile Sabatier, "Compétence plurilingue en contexte formel d'immersion (FL2) et réflexion curriculaire sur la formation des enseignants de français : quand la recherche se met au service de la salle de classe ", Le Journal de l'Immersion / Immersion Journal, vol. 31, n 3, 2009; Diane Dagenais, Danièle Moore, Cécile Sabatier, Patricia Lamarre et Françoise Armand, "Linguistic Landscape and Language Awareness ", dans Durk Gorter et Elana Shohamy (dir.), Linguistic Landscape: Expanding The Scenery, LEA Routledge, 2009, p. 253-269; Cécile Sabatier, "La puce à l'oreille". Représentations d'apprenants sur des sonorités langagières plus ou moins familières ", Revue de l'AQEFLS, numéro spécial "Enseignement et acquisition de la phonétique en classe de langue seconde et étrangère ", 2009, p. 112-133; Diane Dagenais, Françoise Armand, Erica Maraillet et Nathalie Walsh, "L'Éveil aux langues et la co-construction de connaissances sur la diversité linguistique, Revue canadienne de linguistique appliquée, vol. 10, $\mathrm{n}^{\circ} 2$, 2007, p. 197-219. 
développent au quotidien sont au cœur des réflexions qui portent sur la manière de penser aujourd'hui la complexité qui découle de ces situations de multilinguisme.

Alors que l'emploi du terme multilinguisme réfere à une vision situationnelle des contacts de langues qui envisage ces derniers d'un point de vue sociétal, au fil de cet article, nous allons lui préférer le terme de plurilinguisme, en nous centrant plus spécifiquement sur l'individu, point focus et central du contact interlinguistique. Ce déplacement lexical traduit un ancrage conceptuel qui situe l'étude des paramètres fondamentaux des contacts interlinguistiques dans une conception dynamique et multidimensionnelle des locuteurs, attirant par là l'attention sur les valeurs pragmatiques, idéologiques, mais aussi identitaires que ces derniers attachent aux langues.

Au delà des schèmes de compréhension habituels, dans une configuration contextuelle et sociolinguistique du développement des plurilinguismes individuels, il s'agira donc, comme Morin ${ }^{2}$ l'a suggéré, "de s'exercer à une pensée capable de traiter avec le réel, de dialoguer avec lui, de négocier avec lui " pour tenter de répondre aux questions suivantes : quelles pratiques des individus au contact de plusieurs langues déclarent-ils? Quels discours tiennent-ils sur leur vécu sociolinguistique et le développement de leur plurilinguisme et sur les identités (individuelles, collectives, linguistiques et culturelles) qu'ils développent?

Une fois ancrées les notions opératoires dans une revue de littérature appropriée et énoncée ainsi que la méthodologie qui a permis le recueil des données, l'article s'attachera à mettre à jour la construction et le développement des plurilinguismes individuels au regard 1) des modalités d'acquisition et de transmission des langues, 2) des répertoires verbaux déclarés et de la pluralité des identités que les sujets revendiquent pour 3) s'interroger sur la manière de caractériser les situations complexes de contact. Une conclusion reviendra sur l'ensemble du débat pour contribuer à la réflexion.

2 Edgar Morin, Introduction à la pensée complexe, Paris, Éditions du Seuil, 2005, p. 10. 


\section{Ancrages théoriques et conceptuels}

Nos propos sont informés par les discussions théoriques et les travaux qui ont examiné les questions des relations entre les idiomes en contact, de formes et de statuts différents ${ }^{3}$, en rapport avec celles portant sur la socialisation des individus par le biais du langage en général et des langues en particulier ${ }^{4}$, et avec celles portant sur les représentations des langues en lien avec les notions de bi-/plurilinguisme et d'identités plurilingues.

Située dans le champ de l'action sociale, la revue de littérature permet de poser les jalons d'une approche sociolinguistique des bi/plurilinguismes individuels pour cerner, comprendre et expliciter les phénomènes et processus en jeu dans la constitution et la construction des répertoires verbaux et des identités plurilingues. Ce faisant, les ancrages théoriques et conceptuels mobilisés empruntent à différents paradigmes épistémologiques (ceux de la sociologie, de la sociolinguistique, de l'anthropologie linguistique ou de l'ethnolinguistique, entre autres), mais sont partie prenante des études qui portent leur regard sur les locuteurs et

3 Uriel Weinreich, "Unilinguisme et multilinguisme ", dans André Martinet (dir.), Le Langage. Encyclopédie de la Pléiade, Paris, Éditions Gallimard, 1968, p. 647-683; Shana Poplack, "Sometimes I'll start a sentence in Spanish $y$ termino en español ", Linguistics, 18, 1980, p. 581-618; Georges Lüdi et Bernard Py, Etre bilingue, Berne, Peter Lang, 1986; François Grosjean, «The bilingual's language modes ", dans Janet Nicol (dir.), One Mind Two Languages, Oxford, Blackwell, 2001, p. 1-22.

4 Bambi Schieffelin et Elinor Ochs, "Language socialization ", Annual Review of Anthropology, vol. 15, 1986, p. 163-191; Lesley Milroy et Wei Li, «A social network approach to code-switching ", dans Lesley Milroy et Pieter Mysken (dir.), One Speaker Two Languages: Cross-disciplinary perspectives on codeswitching, Cambridge, Cambridge University Press, 1995, p. 136-157.

5 Bernard Py, "Migration, apprentissage et réorganisation des compétences linguistiques ", dans Georges Lüdi et Bernard Py (dir.), Changement de langage et langage du changement. Aspects linguistiques de la migration interne en Suisse, Lausanne, L'Âge d'Homme, 1995, p. 117-146; Georges Lüdi et Bernard Py, Changement de langage et..., op. cit.; Ben Rampton, Crossing: Language and Ethnicity among Adolescents, London, Longman, 1995; Danièle Moore et Claudine Brohy, «Identités plurilingues, dans Sociolinguistique des langues en contact, modèles, théories. Dictionnaire encyclopédique des termes et concepts, Lyon, Ens-Éditions (à paraitre). 
sur le fonctionnement et la circulation des langues en usage en situations complexes ${ }^{6}$.

\subsection{Contacts de langues, pratiques langagières et représentations}

Les premières notions opératoires nécessaires à la compréhension de l'objet d'étude sont celles qui ont trait aux contacts de langues, aux pratiques langagières (réelles ou déclarées) et aux représentations des langues en contact et de leurs locuteurs.

L'expression " contacts de langues " offre la possibilité d'évoquer puis de caractériser les modalités et les types d'alternances de langues rencontrés dans des situations linguistiques de plus en plus complexes :

en dépit de l'importance et de la fréquence des situations plurilingues, il y a une tendance courante, parmi les linguistes même, à considérer l'unilinguisme comme la règle et le plurilinguisme comme quelque chose d'exceptionnel. Cette vision de la réalité, si fortement empreinte d'idéalisme (...) nous fait perdre de vue que des millions d'individus, et peut-être bien la majorité des hommes sur terre, acquièrent le contrôle de plus d'un système linguistique pendant leur vie et emploient, d'une manière plus ou moins indépendante, chaque système selon les nécessités du moment.

Posant d'emblée le plurilinguisme comme règle, l'expression autorise des perspectives d'interprétation qui envisagent les situations sociolinguistiques, soit par un prisme bilinguiste au sein duquel les idiomes en contact sont perçus dans une configuration interactionnelle, microsociolinguistique, synchronique,

6 Danièle Moore, Plurilinguismes et école, Paris, Didier, 2006; Christine Deprez,

Les enfants bilingues : langues et familles, Paris, Crédif Didier, 1994; Christine Deprez, "Pour une conception plus circulante des langues mises en jeu dans les migrations ", dans Louis-Jean Calvet et Auguste Moussirou-Mouyama (dir.), Le plurilinguisme urbain, Paris, Institut de la Francophonie, Didier Érudition, 2000, p. 55-67; Patricia Lamarre et Diane Dagenais, "Trilingual youth in Canada ", dans Charlotte Hoffmann et Jehannes Ytsma (dir.), Trilingualism in Family, School and Community, Clevedon, Multilingual Matters, 2004, p. 53-74; Fabienne Leconte et Clara Mortamet, "La construction des identités plurilingues d'adolescents nouvellement arrivés en France ", dans Pierre Martinez, Danièle Moore et Valérie Spaëth (dir.), Plurilinguismes et enseignement. Identités en construction, Paris, Riveneuve Éditions, 2008, p. 167-177.

7 Uriel Weinreich, op. cit., p. 648. 
dynamique et consensuelle, soit par un prisme diglossique, où la vision de la rencontre entre les idiomes est appréhendée en termes macro-sociolinguistiques, diachroniques, conflictuels et concurrents $^{8}$.

Au regard de notre positionnement qui envisage l'individu comme le lieu du contact, et afin de rendre compte de la complexité de la réalité linguistique qu'il vit, une focalisation au moyen du linguistique sur un point nodal où se cristallisent des conflits d'ordre davantage idéologique vient à omettre qu'il y a, avant tout, rencontre qui n'exclut ni n'impose l'idée de la confrontation dans un contexte donné. Notre conception des contacts de langues repose donc sur une construction de la réalité bi-/plurilingue par des acteurs qui sont au centre des événements langagiers quotidiens : les langues entrent en contact par l'entremise des locuteurs eux-mêmes, lesquels médiatisent ensuite par les pratiques langagières et les usages qu'ils en font les relations langagières : "Les individus membres d'une société sont vus comme étant des agents actifs qui créent leurs propres sens à travers l'emploi stratégique de la langue " ". Cette perspective permet non seulement de ne pas nier les dimensions idéologiques, économiques, et politiques des contacts de langues, mais surtout de les interpréter en tenant compte des différentes dimensions explicatives facilitant l'interprétation des paramètres situationnels et contextuels : une dimension systémique qui rend compte de la rencontre des systèmes linguistiques différents; une dimension sociolinguistique qui souligne l'importance des appartenances individuelles, sociales, politiques, mais aussi culturelles; une dimension interactionniste qui révèle les savoir-faire langagiers

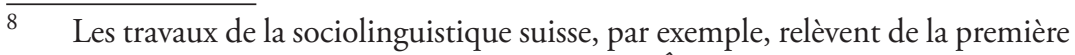
perspective (Georges Lüdi et Bernard Py, Etre bilingue, op. cit.; François Grosjean, op. cit.), tandis que ceux de la sociolinguistique catalane et occitane embrassent la seconde (Henri Boyer, Langues en conflit. Études sociolinguistiques, Paris, L'Harmattan, 1991; Henri Boyer, Plurilinguisme : "contact " ou " conflit " de langues? Paris, L'Harmattan, 1997).

9 Marilyn Martin-Jones, "Les modèles sociolinguistiques dans l'étude du bilinguisme : le cas des minorités linguistiques ", intervention dans le cadre du colloque, Contacts de Langues - Quels Modèles?, Nice, 28-30 septembre 1987, Paris, Maison des Sciences de l'Homme p. 13. 
des locuteurs engagés dans des interactions; et une dimension psycholinguistique qui renvoie aux aspects cognitifs, acquisitionnels mais également affectifs éprouvés par les locuteurs au contact de différents idiomes. Ces dernières dimensions permettent de réincarner les contacts de langues en réintroduisant le sujet au cœur de la réflexion pour ne pas réduire la problématique à une approche purement linguistique.

En prenant comme point focal les rapports (réels, déclarés et représentés) aux langues desdits sujets, les notions de pratiques langagières et de représentations des langues semblent alors contribuer précisément à médiatiser les contacts linguistiques, en en situant socialement les objets et les enjeux dans une dimension et un cadre conceptuels fondés d'une part, sur l'articulation de l'individu et du social (Bourdieu, 1982) et d'autre part, sur des définitions du langage, des locuteurs et de l'activité langagière comme fait social (Bautier, 1995; Billiez, 1997; Melliani, $1999 a^{10}$.

En ce sens, les pratiques langagières des individus inscrivent irrémédiablement le langagier comme fait constitutif d'une dimension sociale où les individus sont considérés comme des agents sociaux marqués par une histoire et des trajectoires de vie personnelles qui agissent sur et dans les situations, et sont ancrées dans les modes de socialisation familiale, amicale ou communautaire, au sein desquels évoluent les individus. Ces modes de socialisation soutenus par des valeurs, des attitudes, des représentations et des usages du et sur le langage, rendent dès lors les pratiques langagières des sujets dépendantes des relations que ces mêmes locuteurs entretiennent avec lesdits idiomes en lien avec l'ensemble des structures du système social, qui exerce sur les membres de la communauté certaines contraintes tout en déterminant les appartenances, les places et les rôles au sein du groupe $^{11}$. Les comportements sociaux et linguistiques sont alors

$\overline{10} \quad$ Cécile Sabatier, Rôle de l'école..., op. cit., p. 149.

11 Dans la lignée des travaux fondateurs variationnistes de Labov $(1976,1978)$, puis fonctionnalistes et interactionnistes de Gumperz (1989) ou encore de Hymes (1984). Voir aussi Steve Marshall, «Langues et identités dans les interactions entre les minorités allochtones et autochtones : essentialisme, appropriation et multiplicité en Catalogne ", dans Pierre Martinez, Danièle Moore et Valérie Spaëth (dir.), Plurilinguismes et enseignement. Identités en 
perçus comme le reflet de l'organisation sociale; ils révèlent les facteurs et les variables qui influencent les choix de codes, les finalités des situations d'interlocution mais aussi les statuts des locuteurs au sein des échanges. La notion de contexte est en conséquence centrale pour réfléchir aux rapports entre positionnements sociaux et identités socioculturelles. Les études en sociologie de l'éducation, mais aussi en sociologie du langage, ou encore en sociolinguistique, insistent désormais sur une approche compréhensive de ces rapports en en pensant les liens avec les circonstances sociales qui les entourent ${ }^{12}$.

Autre facteur essentiel à la compréhension conceptuelle, les représentations sociales, collectives et/ou individuelles, constituent un outil heuristique participant à l'explication de certains comportements linguistiques. Renvoyant à des réalités collectives qui prennent naissance et se développent au sein de groupes sociaux, les représentations sont des approximations du réel, des

systèmes d'interprétation, régissant notre relation au monde et aux autres [qui] orientent et organisent les conduites et les communications sociales, (...) phénomènes cognitifs [qui] engagent l'appartenance sociale des individus par l'intériorisation ${ }_{13}$ de pratiques et d'expériences, de modèles de conduites et de pensée ${ }^{13}$.

Rendue populaire en Europe francophone par les études des psychologues sociaux ${ }^{14}$, sous l'expression de représentations sociales, la notion articulée à celle de contacts de langues permet de porter attention aux valeurs subjectives et sociales accordées aux idiomes en contact. Appréhender les perceptions des locuteurs envers les langues et leurs usages, expliquer certains de leurs comportements linguistiques, et, explorer les images et autres évaluations sociales développées par ces mêmes individus auto-

construction, Paris, Riveneuve Éditions, 2008, p. 77-88; Ben Rampton, op. cit.; Lesley Milroy, Language and Social Networks, $2^{\mathrm{e}}$ édition, Oxford, Blackwell, 1987.

12 Voir, entre autres, Philippe Blanchet, Danièle Moore et Safia Assalah Rahal (dir.), Perspectives pour une didactique des langues contextualisée, Paris, Éditions des Archives Contemporaines et AUF, 2008.

13 Denise Jodelet (dir.), Les représentations sociales, Paris, PUF, 1989, p. 36.

14 Voir, entre autres, Denise Jodelet, op. cit. 
risent en effet à approcher la dynamique interactive de la construction des connaissances et savoir-faire langagiers en situations linguistiques complexes ${ }^{15}$, et, par delà, des répertoires verbaux, car « ces images, le plus souvent fortement stéréotypées, recèlent un pouvoir valorisant ou, a contrario, inhibant (...) $)^{16}$. Participant à interroger les incidences des contacts de langues sur la construction des répertoires verbaux des individus, les représentations, en lien ténu avec les pratiques langagières des sujets, conduisent donc à cerner d'un peu plus près les biographies langagières de ces derniers.

1.2. Biographies langagières, répertoires verbaux et identités plurilingues

La notion de "biographie langagière " renvoie à l'ensemble des expériences linguistiques (sans omettre la dimension culturelle) vécues et accumulées par un individu au cours et à un moment donné de son existence. Ces différentes expériences forment son capital langagier et offrent une vision filigranée de l'acquisition et de l'utilisation des langues en contextes plurilingues. Parce que " les sujets plurilingues construisent dans l'interaction même les termes de leur répertoire $[. . .]^{17}$ ", les études portant sur la dynamique à l'œuvre dans la construction du capital linguistique des individus bi-/plurilingues ${ }^{18}$ se sont attachées à observer la mobilisation des ressources linguistiques au regard des situations de communication, mais aussi des interlocuteurs différents desdits

15 Cécile Sabatier, " "La puce à l'oreille”. Représentations... ", op. cit.

16 Véronique Castellotti, D’une langue à d'autres : pratiques et représentations, Rouen, Publications de l'Université de Rouen, 2001, p. 24.

17 Josiane Boutet et Claire Saillard, «Dynamique des répertoires linguistiques dans la migration chinoise ", dans Jacqueline Billiez (dir.), Contacts de langues. Modèles, typologies, interventions, Paris, L'Harmattan, 2003, p. 97-98.

18 Voir Bonny Norton, Identity and Language Learning: Gender, Ethnicity and Social Change, Harlow, Longman, 2000; Lesley Milroy et Pieter Muysken (dir.), One Speaker Two Languages: Cross-Disciplinary Perspectives on CodeSwitching, Cambridge, Cambridge University Press, 1995; Wei Li, Three Generations Two Languages One Family: Language Choice and Language Shift in a Chinese Community in Britain, Clevedon, Multilingual Matters, 1994. 
individus, des objectifs et des thèmes de communication. Connaître qui parle quelle(s) langue(s), à qui, et quand ${ }^{19}$ conduit à dégager l'ensemble des variétés linguistiques (formelles et informelles) que possède un individu et qu'il met en œuvre selon ses besoins de communication.

La constitution de ce répertoire verbal présente alors des réalités langagières qui renvoient tout à la fois aux potentialités individuelles quant aux choix d'utiliser tel ou tel idiome ou variété de langue, mais aussi aux langues revendiquées comme éléments constitutifs des identités. Entités dynamiques par définition, les répertoires verbaux des individus plurilingues apparaissent d'emblée uniques, mais complexes, pluriels et composites de par les composantes linguistiques et l'éventail des possibilités langagières qu'ils offrent ${ }^{20}$. La conception de l'unicité des répertoires verbaux, à partir de la pluralité des variétés linguistiques qui les composent, s'inscrit dans l'unicité du sujet pensé dans la pluralité de ses pratiques langagières, mais aussi dans la pluralité de ses facettes identitaires qui assurent la construction d'identités marquées par la pluralité des appartenances, des modes d'allégeance aux groupes d'appartenance et de référence $^{21}$. Ces derniers réferent aux instances de socialisation dans lesquels les individus sont inscrits. L'étude des accointances interpersonnelles met à jour les réseaux sociaux $x^{22}$ qui renvoient à la somme des relations, en termes d'interactions linguistiques et sociales, d'un individu avec d'autres personnes et la manière dont ce dernier se positionne à l'intérieur de ces entités sociogéographiques. L'importance des interactions qui y prennent place, en termes de contenu (négociations d'informations, échanges formels, informels, etc.), de réciprocité, de fréquence et de durée, le type de liens qui unit les individus (uniplexe, multiplexe ou

19 Pour paraphraser le titre d'un article fondateur de la sociolinguistique nordaméricaine contemporaine (Fishman, 1965).

20 Georges Lüdi et Bernard Py, Etre bilingue, op. cit.; Danièle Moore, Plurilinguisme et école, op. cit.

21 Cécile Sabatier, «Appartenances identitaires de jeunes Français-Maghrébins à l'école élémentaire française. Entre maux et pratiques ", Child Heath Education / Santé et éducation de l'enfance, Revue en ligne (à paraître).

22 Lesley Milroy, op. cit. 
passif) ainsi que le rôle et la place (centrale ou marginale) des personnes impliquées par rapport à l'individu, sont autant d'indices qui participent à la compréhension de la construction des bi-/plurilinguismes individuels.

Dégager ces réseaux sociaux et linguistiques conduit enfin à mettre en lumière les corrélations entre, d'une part, les pratiques langagières vécues au quotidien et, d'autre part, les identités que les individus plurilingues déclarent. Ce lien manifeste fait dès lors des répertoires verbaux les reflets et les vecteurs des valeurs identitaires individuelles, le sujet parlant utilisant le discours pour négocier sa position sociale dans le groupe.

Parce que l'identité des sujets est sans cesse (ré)ajustée et (re)négociée, dans et par les discours, sa construction se traduit par des figures identitaires distinctes, complexes, parfois contradictoires, voire ambiguës, que l'on peut définir comme des produits contextuels de l'interaction permanente qui se dessine entre les représentations de soi que développent les individus et les représentations sociales, plus ou moins intériorisées par ces derniers ${ }^{23}$.

La relation intrinsèque entre langage et identité conduit par conséquent à concevoir les identités individuelles comme dynamiques, complexes, diffuses ${ }^{24}$ et plurielles. Cette lecture socioconstructiviste du concept envisage dès lors la construction identitaire comme un processus évolutif, non figé, qui varie au gré des relations des individus selon des critères complexes, parfois antagonistes et dépendants des situations d'interactions ${ }^{25}$. En lien avec l'unicité des répertoires verbaux individuels dans la pluralité des pratiques langagières, les " je " successifs d'un individu au contact de plusieurs idiomes apparaissent au fil des discours, à travers les différentes facettes identitaires qu'il laisse émerger.

23 Cécile Sabatier, "Figures identitaires des élèves issus de la migration maghrébine à l'école élémentaire en France ", Éducation et Francophonie, vol. 34, nº 1, 2006a, p. 116.

24 Georges Lüdi et Bernard Py, Changement de langage..., op. cit.

25 Jean-Claude Kaufmann, L'invention de Soi. Une théorie de l'identité, Paris, Hachette, 2004. 


\subsection{Plurilinguisme, acquisition et transmission des langues}

Les dernières notions opératoires pour la définition de l'objet d'étude sont des notions transversales plus génériques qui nous positionnent dans le champ des travaux portant sur l'acquisition et la transmission des langues dans des contextes de développement plurilingue. L'exploration des contacts de langues et de la construction des plurilinguismes individuels appelle en effet à s'interroger sur les modalités d'acquisition et de transmission des langues en situations linguistiques complexes, dans la mesure où l'analyse des différents mécanismes à l'œuvre met à jour les incidences du contact sur le développement cognitif et linguistique de l'individu en termes d'aptitudes et d'affectivité ${ }^{26}$.

$\mathrm{Au}$ regard des théories générales d'acquisition du langage, l'étude des contacts de langues, telle que nous la mobilisons ici, s'inscrit dans une perspective interactionnelle et constructiviste qui repose sur l'approche vygotskyenne de la cognition. Cette conception interactionniste ancre, là encore, la dimension fonctionnelle du langage dans les contextes linguistiques, cognitifs et sociaux. Les structures langagières n'étant ni innées ni acquises, mais résultant de l'interaction entre le développement cognitif de l'individu et l'environnement sociolinguistique, le rôle de l'entourage familial ainsi que l'influence du milieu social sont constitutifs de la genèse de l'apprentissage linguistique. Ainsi, l'étude des phénomènes de contacts de langues (et par voie de conséquence de bi-/plurilinguisme) ne peut que contribuer à une meilleure compréhension et connaissance des mécanismes en jeu et à l'œuvre dans l'acquisition des langues secondes ou tierces; et inversement la mobilisation des connaissances sur l'acquisition des langues secondes ou tierces permet, elle, d'interroger, de connaître puis de comprendre les mécanismes (psychologiques,

26 Voir, entre autres, Paul Boggards, Aptitude et affectivité dans l'apprentissage des langues étrangères, Paris, Crédif / Hatier, 1988; Aneta Pavlenko et Adrian Blackledge, Negotiation of Identities in Multilingual Contexts, Clevedon, Multilingual Matters, 2004; Cécile Sabatier, "La puce à l'oreille". Représentations... ", op. cit. 
cognitifs et affectifs) à l'œuvre dans le développement individuel du bi-/plurilinguisme ${ }^{27}$.

Ainsi, en lien direct avec l'acquisition des langues en contexte de contacts linguistiques au sein de réseaux sociaux et linguistiques complexes, apparaît dans le cadre de l'interrogation qui entoure la construction et le développement des répertoires langagiers plurilingues, le champ conceptuel de la transmission des idiomes et plus spécifiquement les notions de statuts et de fonctions assignés aux langues. Ces notions participent en effet à caractériser la manière dont des individus en contact avec plusieurs langues sont amenés à accorder des valeurs différenciées à ces idiomes en présence, au regard du capital économique, linguistique et affectif qu' ils leur attribuent ${ }^{28}$. Ce faisant, conjointement à une analyse factorielle de variables " extra-linguistiques ", il paraît opportun de chercher les statuts et les fonctions sociales dévolues aux langues qui composent les répertoires verbaux individuels, dans la mesure où se dessine chez les individus bi-/ plurilingues, une répartition des codes fluctuante selon différents paramètres situationnels et fonctionnels qui permettent de mettre en lumière les buts ou les intentions que ces locuteurs reconnaissent à leurs conduites langagières.

Les fonctions ne sont pas attachées de façon immuable à des langues particulières. Ce sont, au contraire, des conjonctions, des contrastes et des complémentarités entre les fonctions des diverses langues qui forment les répertoires verbaux des locuteurs ${ }^{29}$.

À travers les statuts et les fonctions attribués aux langues en contact, s'esquisse une gestion des interfaces qui met en lumière le lien existant entre la valorisation subjective de tous les codes et leurs utilisations effectives et qui participe à rendre explicite la

\footnotetext{
$\overline{27}$ Philip Herdina et Ulrike Jessner, A Dynamic Model of Multilingualism Perspectives of Change in Psycholinguistics, Clevedon, Multilingual Matters, 2002; Ellen Bialystok, Bilingualism in Development. Language, Literacy and Cognition, Cambridge, Cambridge University Press, 2001. Patricia Lamarre et Diane Dagenais, op. cit.

29 Jacqueline Billiez (coord.), Une semaine dans la vie plurilingue à Grenoble, Observatoire des pratiques linguistiques, DGLF, Grenoble, Lidilem (non publié), 2000, p. 50.
} 
construction des plurilinguismes individuels. Calvet ${ }^{30}$ a proposé un modèle gravitationnel du contact des langues pour représenter les liens d'attraction ou de répulsion entre idiomes et locuteurs. Ce modèle tend à visualiser graphiquement l'organisation des plurilinguismes en cherchant à dégager pour l'individu en situation linguistique complexe les idiomes qui occupent une place centrale et ceux plus périphériques dans sa biographie langagière.

Ces liens de gravitation entre les langues abritent toute une série d'espaces, réels, symboliques ou imaginés, au sein desquels se dessinent et se négocient des identités locales, nationales, et transnationales ${ }^{31}$.

L'étude de la socialisation linguistique des locuteurs plurilingues, à partir des réseaux sociolinguistiques qu'ils déclarent et à travers les statuts et les fonctions attribués aux idiomes en présence, est donc clé pour expliquer et justifier la présence de tel ou tel idiome dans les répertoires plurilingues, pour explorer les liens avec l'environnement contextuel, ainsi que pour entrevoir des répercussions sur la distribution des langues et sur leurs fonctions, et donc pour comprendre l'appropriation et la transmission des langues en situations linguistiques complexes.

\section{Méthodologie}

Explorer puis comprendre les processus, mais aussi les facteurs à l'œuvre dans la construction et le développement des plurilinguismes individuels par le biais d'un regard porté aux pratiques langagières et aux répertoires verbaux des individus en situation de contacts de langue, conduit en termes méthodologiques à solliciter des outils de recherche qui adoptent une démarche permettant de saisir les langues des individus, les discours qu'ils tiennent sur ces dernières, mais aussi leurs comportements et leurs représentations vis-à-vis de celles-ci dans une méthodologie qui n'abstrait pas les individus et leurs langues de la micro-société dans laquelle ils vivent et fonctionnent. L'étude empirique des

\footnotetext{
30 Louis-Jean Calvet, Pour une écologie des langues du monde, Paris, Plon, 1999, p. 77.

31 Danièle Moore, op. cit., p. 29.
} 
bi-/plurilinguismes individuels relève de "l'ensemble de [ces] facteurs situationnels qui se révèlent pertinents pour la compréhension d'un comportement langagier ${ }^{32}$ ». Dégager les traits contextuels individuels qui peuvent expliquer la construction du bi-/plurilinguisme chez les sujets de l'enquête, décrire leurs usages des langues dans différents contextes sociaux, familiaux, scolaires et expliquer les relations qui se tissent entre les phénomènes langagiers, d'une part, et les constructions des identités en contexte plurilingue, d'autre part, participent à accéder à cette compréhension sans soustraire les individus de la micro-société dans laquelle ils évoluent.

Comme l'étude des pratiques langagières individuelles n'a de sens que mise en relation avec celle des représentations vis-à-vis des idiomes en contact et de leurs locuteurs, la mise en évidence des univers de significations s'ancre dans une approche compréhensive des répertoires verbaux déclarés par les individus plurilingues afin de caractériser l'objet du savoir. Cette approche méthodologique vise à comprendre, à décrire, à expliquer et à interpréter les phénomènes dans leurs relations à la totalité de l'expérience des sujets et nous ancrent dans une logique émique à la jonction de l'individu et du social. Donner à voir les logiques des situations et celles des représentations pour saisir la manière dont elles s'articulent chez et pour l'individu prend également appui sur des entretiens compréhensifs ${ }^{33}$ à orientation semidirective qui permettent d'approfondir les rapports des individus aux langues et cultures en contact, à leurs appartenances identitaires, mais également aux usages, aux fonctions et aux autres valeurs qu'ils attribuent aux différents idiomes qui composent leurs répertoires verbaux.

32 Georges Lüdi et Bernard Py, Changement de langage..., op. cit., p. 14.

33 Les thématiques du guide d'entretien étaient les suivantes : auto-présentation du sujet, langues en contact dans les répertoires verbaux, usages et pratiques, langues et identités, mélange des langues et plurilinguisme, compétences langagières et (in)sécurité linguistique, choix linguistiques et transmission linguistique, langue arabe et école, environnement. Voir Jean-Claude Kaufmann, L'entretien compréhensif, Paris, Nathan Université, 1996. 
Onze entretiens compréhensifs sociobiographiques d'environ 90 minutes chacun ont ainsi été menés et réalisés en 2000 et 2001 auprès de jeunes individus plurilingues dans le cadre d'un travail qui s'est attaché au rôle de l'école dans le développement et la construction du plurilinguisme chez des enfants issus de la migration maghrébine en France ${ }^{34}$. Ces jeunes plurilingues, issus des communautés algérienne, marocaine et tunisienne, plus que toute autre communauté allogène en France, sont affublés de ce que Kaufmann ${ }^{35}$ appelle " un capital d'images entamé d'emblée par un codage négatif ». Ils ne sont pas perçus dans les représentations collectives comme des individus (potentiellement) bi-/ plurilingues et, les langues arabes et leurs pratiques sont parmi les plus dévalorisées socialement. Étudier la construction des plurilinguismes individuels dans ces situations sociales nous semble particulièrement révélateur des contextes, des enjeux, mais aussi des facteurs à l'œuvre favorisant et/ou inhibant le développement plurilingue.

Ces jeunes d'origine maghrébine ( 5 fillettes et 6 garçons) sont nés en France. Âgés de 9 à 11 ans, issus d'un milieu socio-économique ouvrier, ils viennent de familles nucléaires, à l'exception de l'un d'entre eux qui vit seul avec sa mère. Tous appartiennent à des fratries plus ou moins nombreuses; leur position au sein des fratries varie de benjamins à cadets. Ils ont donc tous des frères et sœurs aînés pouvant servir de modèles (en positif ou négatif) dans la construction d'identités d'appartenance et euxmêmes peuvent servir de modèles pour leurs frères et sœurs plus petits. Leurs parents occupent des catégories socioprofessionnelles relativement semblables. La majorité des parents des jeunes interviewés en 2001 (ou au moins l'un d'entre eux, et essentiellement les mères) sont nés en France, et les jeunes ont déclaré la présence de leurs grands-parents sur ce même sol français; contrairement aux jeunes rencontrés en 2000 qui ont majoritairement déclaré des parents nés sur le sol algérien (tous les jeunes de cette année-là étant issus de familles d'origine algérienne) et

\footnotetext{
34 Cécile Sabatier, Rôle de l'école..., op. cit.

35 Jean-Claude Kaufmann, L'invention de Soi..., op. cit., p. 212.
} 
des grands-parents y résidant encore. Les lieux de naissance des parents et le maintien des liens avec des membres de la famille se révèleront un facteur explicatif important dans l'analyse des modalités de transmission des langues et des constructions identitaires de ces jeunes.

Les tableaux synoptiques ci-après présentent en détails les participants de l'étude. Pour protéger l'anonymat des participants, seule l'initiale de leur prénom suivie de l'année de recueil des données est utilisée.

\section{Tableau 1}

\section{Présentation des participants - Échantillon année 2000}

\begin{tabular}{|l|c|c|c|l|l|}
\hline Sujets & Sexe & $\begin{array}{c}\text { Année de } \\
\text { naissance }\end{array}$ & $\begin{array}{c}\text { Lieu de } \\
\text { naissance }\end{array}$ & \multicolumn{1}{|c|}{ Situation familiale } & $\begin{array}{l}\text { Pays d'origine } \\
\text { de la famille }\end{array}$ \\
\hline Ar-00 & $\mathrm{M}$ & 1989 & France & $\begin{array}{l}4 \text { frères et sœurs } \\
\text { Ne connaît pas le lieu de } \\
\text { naissance de ses parents } \\
\text { 2 grands-mères en France }\end{array}$ & $\begin{array}{l}\text { Algérie } \\
\text { (séjours } \\
\text { estivaux } \\
\text { annuels) }\end{array}$ \\
\hline Me-00 & F & 1988 & France & $\begin{array}{l}\text { 4 frères et sœurs } \\
\text { Parents nés en Algérie } \\
1 \text { grand-mère en France }\end{array}$ & $\begin{array}{l}\text { Algérie } \\
\text { (séjours } \\
\text { estivaux tous les } \\
\text { deux ans) }\end{array}$ \\
\hline N-00 & M & 1989 & France & $\begin{array}{l}\text { Frères et sœurs (n'a pas précisé } \\
\text { le nombre) } \\
\text { Parents nés en Algérie } \\
\text { Grand-mère maternelle en } \\
\text { France et Grand-père maternel } \\
\text { en aller/retour entre France et } \\
\text { Algérie }\end{array}$ & $\begin{array}{l}\text { Algérie } \\
\text { (séjours } \\
\text { estivaux } \\
\text { annuels) }\end{array}$ \\
\hline S-00 & M & 1988 & France & $\begin{array}{l}1 \text { sœur } \\
\text { Mère née en France } \\
1 \text { grand-mère en France }\end{array}$ & $\begin{array}{l}\text { Algérie } \\
\text { (n'est jamais } \\
\text { allé en Algérie) }\end{array}$ \\
\hline Y-00 & M & 1989 & France & $\begin{array}{l}5 \text { frères et 2 sœurs en Algérie } \\
\text { Parents nés en Algérie } \\
\text { Père absent du foyer } \\
\text { Grands-parents en Algérie }\end{array}$ & $\begin{array}{l}\text { Algérie } \\
\text { (séjours } \\
\text { estivaux } \\
\text { annuels) }\end{array}$ \\
\hline
\end{tabular}


Tableau 2

Présentation des participants - Échantillon année 2001

\begin{tabular}{|c|c|c|c|l|l|}
\hline Sujets & Sexe & $\begin{array}{l}\text { Année de } \\
\text { naissance }\end{array}$ & $\begin{array}{c}\text { Lieu de } \\
\text { naissance }\end{array}$ & \multicolumn{1}{|c|}{ Situation familiale } & \multicolumn{1}{|c|}{$\begin{array}{c}\text { Pays d'origine } \\
\text { de la famille }\end{array}$} \\
\hline A-01 & F & 1990 & France & $\begin{array}{l}\text { 4 frères et sœurs } \\
\text { Parents nés en Algérie } \\
1 \text { grand-mère en France }\end{array}$ & $\begin{array}{l}\text { Algérie } \\
\text { (séjours estivaux } \\
\text { annuels) }\end{array}$ \\
\hline D-01 & F & 1990 & France & $\begin{array}{l}\text { Frères et sœurs (n'a pas précisé } \\
\text { le nombre) } \\
\text { Parents nés en France } \\
\text { 2 grands-mères en France }\end{array}$ & $\begin{array}{l}\text { Algérie } \\
\text { (n'est jamais allée en } \\
\text { Algérie - uniquement } \\
\text { son père) }\end{array}$ \\
\hline H-01 & M & 1990 & France & $\begin{array}{l}\text { Frères (n'a pas précisé le nombre) } \\
\text { Mère née en France; n'est pas } \\
\text { très sûr du lieu de naissance de } \\
\text { son père (Algérie ou Maroc) } \\
\text { Grands-parents en France }\end{array}$ & $\begin{array}{l}\text { Algérie } \\
\text { (n'est jamais allé en } \\
\text { Algérie) }\end{array}$ \\
\hline K-01 & F & 1989 & France & $\begin{array}{l}\text { 6 frères et sœurs } \\
\text { Parents nés au Maroc } \\
\text { Grands-parents au Maroc }\end{array}$ & $\begin{array}{l}\text { Maroc } \\
\text { (séjours estivaux - } \\
\text { aucune information sur } \\
\text { fréquence) }\end{array}$ \\
\hline
\end{tabular}

Les données, recueillies sur magnétophone et transcrites dans leur intégralité, ont fait l'objet d'une analyse de discours et de contenus. Cette dernière a mis à jour des aspects langagiers et culturels des jeunes en exposant les articulations entre leurs attitudes et représentations face aux langues et cultures en contact, en particulier face à la langue et à la culture arabes, socialement et scolairement minorées, et les pratiques linguistiques et culturelles qu'ils déclarent. Elle a également mis en lumière des récits d'expérience qui ont dévoilé différents positionnements identitaires en lien avec les contextes dans lesquels ils se déploient, et dépassant les frontières d'une identité ancrée dans une seule appartenance. Enfin, au regard de la question de transmission des langues au sein des instances de socialisation, il est apparu que pour ces jeunes, transmission n'était pas synonyme nécessairement de conservation en l'état ou de reproduction à l'identique. 


\section{Résultats, analyse et discussion}

\subsection{Mise en lieux des répertoires verbaux plurilingues}

Létude des pratiques déclarées met en lumière chez les onze sujets participants, en plus des langues qui composent les répertoires verbaux, la manière dont ils utilisent ces dernières au regard des situations de communication dans lesquelles ils sont engagés. L'alternance des langues en présence qu'ils déclarent mobiliser selon leurs réseaux sociolinguistiques révèle l'existence de différentes modalités de contact qui permettent d'envisager les divers lieux de médiation linguistique ainsi que les rôles de chacun des locuteurs en présence.

Les discours des jeunes font apparaître une dépolarisation des profils langagiers (lesquels ne se réduisent pas aux seules langues française et arabe) et les instances de socialisation clés pour le développement des réseaux linguistiques et sociaux : la famille, l'école et la communaute ${ }^{36}$. La multitude de langues ou de formes linguistiques déclarées être utilisées donne à voir un environnement contextuel et relationnel complexe, au sein duquel les jeunes issus des communautés maghrébines évoluent au contact de nombreux idiomes qui ont des statuts et des fonctions diversifiés. Tous les jeunes participants ont ainsi déclaré être en contact avec au minimum trois langues pour Ar-00, H-01, K-01 et D-01) et jusqu'à six pour certains des garçons ( $\mathrm{Y}-00$ et $\mathrm{N}-00$ ). Ils ont également déclaré de manière plus précise mobiliser des compétences de réception avec deux d'entre eux et cinq jeunes (N-00, Y-00, A-01, M-01 et Mo-01) ont souligné qu'ils en utilisaient au moins deux en production de manière quotidienne. À côté, d'une part, du français et de ses divers registres et variétés (verlans, argots enfantins), et, d'autre part, des arabes dialectaux (algérien, marocain, tunisien, berbère ${ }^{37}$ ), ont également été cités

\footnotetext{
36 Charlotte Hoffmann et Jehannes Ytsma (dir.), Trilingualism in Family, School and Community, Clevedon, Multilingual Matters, 2004.

37 Le terme « berbère " fait directement allusion à la situation linguistique du Maghreb (évoquée par les jeunes sujets de l'étude) et renvoie à un groupe d'idiomes qui comprend le chaoui, parlé dans le massif des Aurès en Algérie, le kabyle parlé en Kabylie et trois autres variantes parlées au Maroc : le
} 
l'italien, langue apprise à l'école ${ }^{38}$, l'anglais, langue hypercentrale dont chacun connaît l'utilité instrumentale et le statut valorisé, mais aussi le polonais, le néerlandais, le créole martiniquais. Ces langues vernaculaires sont présentes dans les quartiers où vivent les jeunes; elles apparaissent dans les répertoires verbaux des intéressés par le biais des réseaux de pairs au contact desquels les participants évoluent :

522/A-01 : alors avec y a des amies qui sont Martiniquaises, alors des fois, elles nous parlent un petit peu, puis ça elles nous apprennent quelques mots, donc alors donc nous on se parle tous un petit peu des langues qu'on a, et puis voilà (EntEl-EntA01).

Cet ensemble de variétés linguistiques traduit non seulement la richesse et la diversité de l'environnement social urbain, linguistique et culturel, mais également il pose les jeunes comme acteurs sociaux, membres actifs d'un ou de plusieurs groupes et constamment engagés dans des (inter)actions collectives. L'exemple de la présence des parlers urbains ${ }^{39}$ dans les répertoires verbaux de certains des jeunes de l'étude témoigne de l'utilisation du langage dans l'espace urbain comme un indice d'urbanité qui met à jour la microsociété adolescente qui se sert des langues pour exprimer son adhésion (ou non) à des valeurs partagées dans lesquelles viennent se fondre les différences pour construire un espace d'interactions sociales qui diverge de la société adulte environnante. L'extrait d'entretien qui suit met ainsi en avant la dynamique qui se joue dans les groupes de pairs pour inclure ou

tamaziht (ou rifrain) parlé au nord du pays, le tashellet (ou chleuh) parlé au sud et le tamasheq parlé par les Touaregs du Sahara.

38 Dans l'école élémentaire fréquentée par les jeunes de l'enquête, conformément aux directives qui imposent l'enseignement d'une langue étrangère dès le grade 4, le choix de l'établissement s'est porté sur la langue italienne. Ce choix reflète la volonté de l'établissement d'inscrire l'enseignementapprentissage des langues étrangères dans le contexte social, linguistique et historique de la ville dans laquelle il se situe. Cette dernière, en effet, de par sa proximité avec la frontière italienne, accueille une très grande population migrante transalpine.

39 Parlers que l'on qualifie aussi de sociolectes générationnels ou de " langages des jeunes ". Voir l'exemple de la langue de f/Ø/ dans Cécile Sabatier, " "La puce à l'oreille. Représentations... ", op. cit. 
exclure les membres et les non-membres et la manière dont les jeunes se positionnent dans ces derniers :

719/Enq : (...) tu peux me décrire l'environnement dans lequel tu vis? (...) tu vis dans une maison, dans un immeuble?

720/M-01 : dans un immeuble, dans un grand quartier 721/Enq : oui à Sourceau ${ }^{40}$ ?

722/M-01 : oui à la Bastille où les jeunes sont vraiment pas polis

723/Enq : oui

724/M-01 : ils font que des tags sur les murs et qui s'insultent

725/Enq : ça te gêne?

726/M-01 : qui se bagarrent beaucoup, et qui volent, et qui mettent les motos qu'ils volent dans les caves, dans les poubelles, et puis que la police, ça me gêne beaucoup qu'elle tourne toujours dans le quartier

727/Enq : ça donne une mauvaise image du quartier 728/M-01 : oui, oui, moi ça me gêne

729/Enq : et avec ces gens du quartier, avec ces jeunes tu utilises quelle(s) langue(s)? tu parles quelle(s) langue(s) avec eux? 730/M-01 : ils parlent vulgaire alors je parle vulgaire (EntEl-EntM01).

Selon M-01, les parlers des jeunes sont à l'image du quartier; la jeune fille associe dans ses propos une image plutôt négative à un parler «vulgaire » qui met en évidence la transmission de pair à pair et la manière dont les jeunes agissent pour être acceptés dans des réseaux d'adolescents en développant des compétences dans les idiomes en usage dans ces groupes. Symboles d'adhésion, d'intégration au groupe et d'identification, les façons de parler au sein des groupes de pairs s'élaborent également sur la base des différentes communautés linguistiques dont sont issus les jeunes issus de familles migrantes :

558/Me-00 : y a des Italiens, y a des Anglais, y a des Néerlandais (EntEl-EntMeO0).

40 Le nom de la ville et de ses quartiers a été modifié pour respecter l'anonymat des participants.

41 Ces propos montrent que le répertoire obscène s'apprend souvent assez jeune, dès l'école primaire, à la fois par initiation réciproque et par imitation. 
Ces groupes de pairs ne sont pas constitués sur une base ethnique stricte, mais sur une base interethnique, expliquant par là même comment la présence dans le quartier d'un pair favorise ou non un enracinement local des idiomes et leur utilisation :

531/Enq : et ici, est-ce que tu as des amis qui ne parlent pas arabe, mais qui vont utiliser quelques mots d'arabe?

532/M-01 : oui, y a ceux de ma classe

533/Enq : oui alors comment ils connaissent ces mots?

534/M-01 : A-01 elle dit plein de petits gros mots en arabe et puis eux ils répètent $(E n t E l-E n t M 01)$.

L'environnement urbain des jeunes est donc un lieu de brassage de vies, d'individus avec des expériences uniques, un lieu de brassages des langues. En s'attachant de plus près aux aires d'emploi des différents idiomes en contact dans les répertoires verbaux des jeunes, à partir de l'étude de la structuration linguistique de l'espace urbain social et scolaire ${ }^{42}$, il apparaît que les jeunes développent une conscience langagière aiguë des statuts et des fonctions des langues en contact ainsi que du rôle qu'ils jouent dans la transmission des langues. Interrogés sur les langues en contact au sein de l'enceinte scolaire, les jeunes ont tous rapporté une structuration spatiale entre " un dedans " et " un dehors " qui fragmente linguistiquement l'espace social et scolaire en zones interstitielles dans lesquelles certains idiomes sont autorisés ou non; mais ils ont également témoigné de la perméabilité des frontières symboliques entre ces espaces a priori clairement délimités. La mise en lieux des langues traduit ainsi leur capacité à porter attention aux formes du langage et à manipuler ces mêmes formes pour s'adapter à la réalité contextuelle de la situation de communication. L'interpénétration des idiomes met en avant une conception dynamique et circulante des langues, laquelle se manifeste encore plus explicitement dans les pratiques langagières familiales :

$\overline{42}$ Cécile Sabatier, «Symbolique des lieux et structuration linguistique de l'espace scolaire : comment les élèves redessinent les frontières de langues à l'école ", dans Christine Hélot (dir.), Écarts de langues, écarts de cultures. À l'école de l'Autre, Berne, Peter Lang, 2006b, p. 75-86. 
28/N-00 : (...) ça dépend où on est, si on est à la maison, il [mon père] va parler plus arabe, mais si y a des copains avec moi, il va parler français

29/Enq : et toi tu lui réponds en quelle(s) langue(s)?

30/N-00 : français ou arabe, ça dépend si on est entre nous, je parlerai arabe

31/Enq : oui et si lui te parle en français, tu vas lui répondre en quelle(s) langue(s)? en arabe?

32/N-00 : français

$33 / \mathrm{Enq}$ : français, et s'il te parle en arabe tu lui réponds en?

34/N-00 : arabe

35/Enq : arabe

36/N-00 : je réponds la langue qu'on te dit

37/Enq : (...) d'accord et avec maman comment ça se passe?

38/N-00 : la même [façon] (EntEl - EntNOO).

Cet extrait souligne certains des paramètres conversationnels qui viennent éclairer davantage la dynamique des langues chez les jeunes et leurs familles. N-00 décrit en effet une répartition fonctionnelle des codes français et arabe liée à une fonction de convergence langagière dépendante du lieu de l'interaction, des interlocuteurs, des niveaux de compétences de ces derniers dans chacune des langues en présence. N-00 souligne également des facteurs plus affectifs dans le recours ou non à telle ou telle langue expliquant par là que le choix de langues s'effectue consciemment pour accepter ou rejeter dans l'échange le parler vernaculaire commun.

L'examen de l'usage des langues en famille chez l'ensemble des jeunes de l'enquête révèle encore plus explicitement les politiques linguistiques en vigueur au sein des familles et le rôle que les jeunes y occupent. La famille apparaît, en effet, comme un lieu de double médiation linguistique entre, d'une part, parents et grands-parents et, d'autre part, parents et enfants. Plus spécifiquement, enfants, parents et grands-parents ont des répertoires verbaux différents, qu'ils parviennent à faire converger à travers l'émergence d'un parler bi-/plurilingue familial unificateur, chacune des parties en présence servant de médiateurs linguistiques dans chacune des langues en présence. Les enfants occupent la fonction de médiateurs linguistiques dans l'acquisition du 
français des parents; ils sont généralement à l'interface de la famille et de la société dès lors qu'il s'agit d'expliquer les codes à leurs aînés. À l'inverse, ce sont les parents, mais surtout les grands-parents (et plus spécifiquement encore les grands-mères) qui maintiennent l'arabe au sein des cellules familiales. La présence langagière de la langue familiale ne va cependant pas toujours de pair avec l'usage effectif de la langue dans la famille, ni même avec la connaissance de cette dernière par les plus jeunes :

4/D-01 : je sais parler le français, c'est tout, mais ma grand-mère elle parle algérien et je le comprends voilà (EntEl-EntD01).

Les discours des jeunes mettent aussi en lumière des continuités et des ruptures dans la transmission des langues qui là encore traduit l'adaptabilité des jeunes aux situations de communication. La transmission pour ces derniers ne passe pas par une conservation en l'état des idiomes familiaux ni même par une reproduction à l'identique ${ }^{43}$; elle repose davantage sur un jeu subtil d'adaptations et de régulation entre les langues en présence pour répondre à de nouveaux besoins langagiers, communicatifs et également identitaires. Les deux exemples schématisés de communication intra-familiale ci-après montrent que le patrimoine linguistique et culturel d'origine des familles issues de l'immigration maghrébine relève d'une dialectique organisée entre des langues reçues en héritage (et que les grands-parents incarnent ou symbolisent selon les niveaux de compétences des jeunes et les degrés de contact avec les familles restées au pays) et celles qui sont utilisées au quotidien dans les pratiques de la famille.

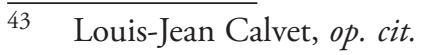




\section{Figure 1}

\section{Communication intrafamiliale chez Mo-01}
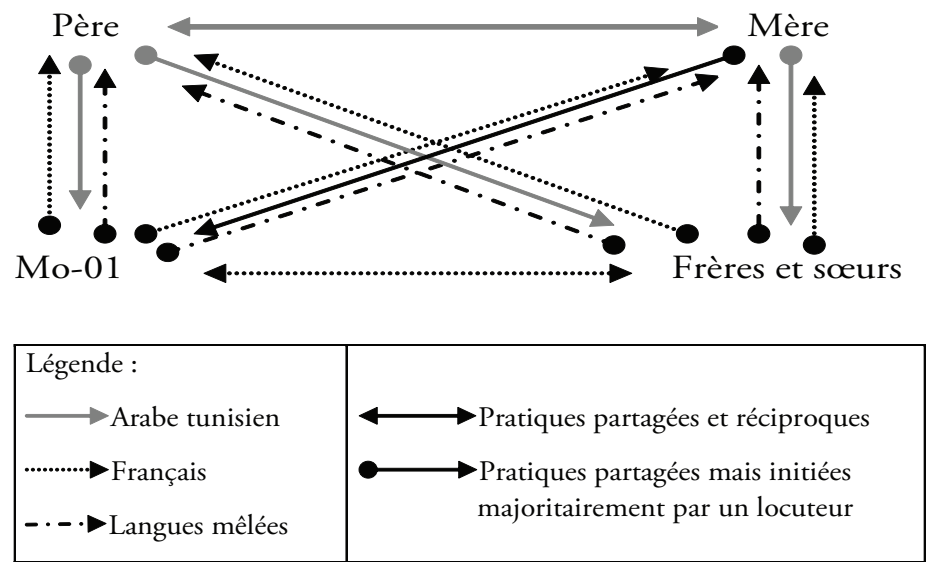

Figure 2

\section{Communication interfamiliale chez D-01}

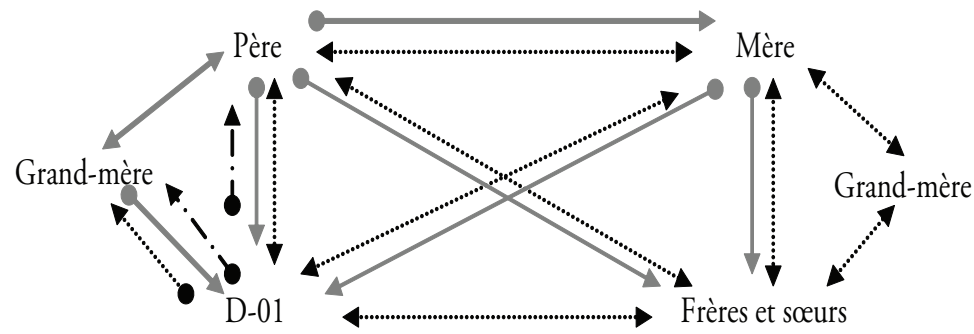

\begin{tabular}{|c|c|}
\hline gende & \\
\hline$\ldots \ldots \ldots . . \cdots$ Français & $\longleftrightarrow$ Pratiques partagées et réciproques \\
\hline $\begin{aligned} \longrightarrow & \text { Arabe algérien } \\
\longrightarrow & \text { Algérien-français : " petits mots » échangés } \\
& \text { par D-01 avec son père et sa grand-mère }\end{aligned}$ & $\begin{array}{l}\longrightarrow \text { Pratiques partagées mais initiées } \\
\text { majoritairement par un locuteur }\end{array}$ \\
\hline
\end{tabular}

\subsection{Des identités plurielles}

L'évocation des pratiques conversationnelles des familles et de certaines des stratégies que les jeunes déploient dans les interactions pour parvenir à maintenir et combler les asymétries de leurs 
répertoires verbaux, les a conduit à parler explicitement de leur plurilinguisme et du lien qui s'établit inéluctablement entre leur parler familial vernaculaire et la construction de leur(s) identité(s). En effet, la grande majorité des interactions rapportées entre enfants, fratries, parents et grands-parents, en France ou dans les pays d'origine des familles lorsqu'un contact est maintenu, fait apparaître un parler vernaculaire intra-familial bi-/plurilingue. Ce parler aux multiples formes linguistiques, dont certaines sont identifiables comme appartenant au français, d'autres à l'arabe, et d'autres, enfin, plus difficilement identifiables entre français et arabe, témoigne des choix de langues effectués par les jeunes bi-/ plurilingues et négociés avec leurs interlocuteurs dans telle ou telle situation de communication. Dans les extraits qui suivent, extraits relevés au fil de l'entretien, Mo-01 explique parfaitement comment ce parler bi-/plurilingue fonctionne :

16/Mo-01 : (...) des fois on parle arabe, mais dès que je comprends pas un mot en arabe et bien ils me le disent en français

110/Mo : (...) dès que je comprends pas un mot en tunisien je leur dis en français

296/Mo-01 : (...) des fois je sais pas comment leur dire alors je leur dis en français

308/Mo-01 : dès que je lui raconte [à ma mère] dès que je commence à parler je parle une langue et dans le milieu je change de langue

318/Mo-01 : (...) et moi je leur parle tunisien et après je change de langue (...)

326/Mo-01 : (...) des fois je parle en français des fois je parle en tunisien

330/Mo-01 : c'est comme ça la double langue (EntEl - EntMoO1).

On voit ici se dégager un parler polyfonctionnel qui marque à la fois l'appartenance de Mo-01 à un groupe, à une famille, voire à une communauté, mais aussi l'ensemble des possibilités expressives dont il dispose pour communiquer. L'évocation de la 
" double langue » verbalise l'ajustement linguistique réciproque qui s'opère entre interlocuteurs. Les deux langues perçues initialement, et pour certains besoins de communication, comme des entités distinctes, n'en forment en fin de compte qu'une seule et unique; cela traduit de fait la représentation que le sujet se fait des frontières entre langues, ou plus précisément, de la perméabilité des frontières entre les langues, qui prennent leur sens dans les représentations des participants. La réorganisation des compétences linguistiques dans les répertoires langagiers, à partir des variantes de français et d'arabes, donne naissance à une nouvelle variante polylectale qui 1) démontre comment les jeunes réinterprètent et se réapproprient les langues familiales à travers le parler bi-/plurilingue, en créant un micro système pluriel, dynamique et instable, et 2) participe à la création de l'expression d'une nouvelle appartenance ou d'affirmations identitaires plurielles.

En effet, par delà l'instance familiale et les pratiques langagières, les jeunes ont fait mention d'une pluralité d'identités qui est le pendant de leur pluralité linguistique et culturelle. À la lisière de plusieurs langues, de plusieurs cultures, mais aussi de plusieurs environnements spatiaux et symboliques, les jeunes ont déclaré déployer des affirmations et des figures identitaires plurielles contextualisées :

220/Mo-01 : les Tunisiens je veux qu'ils me prennent pour un Tunisien, et les Français je veux qu'ils me prennent pour un Français

221/Enq : pourquoi?

222/Mo-01 : je sais pas je veux que tout le monde mapprécie

(...)

296/Mo-01 : y en a qui me connaissent pas, y en a qui me connaissent pas, des fois ils me demandent l'heure, et des fois je sais pas comment dire, alors je leur dis en français, ils me font «toi tu es Français-Tunisien » je fais " oui " (EntEl - EntMo01).

Les propos de Mo-01 soulignent que les jeunes construisent leur(s) identité(s) à travers et par les systèmes linguistiques dans lesquels ils sont socialisés. Cette co-construction interactive des identités en contextes émerge dans et par l'activité des individus 
engagés dans une interaction. Moore rappelle d'ailleurs que « les cadres ainsi définis sont largement (re)construits par les locuteurs (plurilingues - apprenants - acteurs sociaux) dans l'action et dans leurs représentations ${ }^{44}$ ». En procédant à une lecture diachronique de la construction identitaire des jeunes déclarée au fil des entretiens, différentes figures identitaires se sont révélées et ce faisant ont mis à jour les mécanismes à l'œuvre qui permettent aux individus de se distancier de socialisations qui définissent l'individu, mais aussi les processus d'identification et d'identisation des jeunes ${ }^{45}$. Ainsi, trois grandes figures identitaires peuvent être dégagées des discours des onze participants de l'étude. La première "Comme un Arabe " (EntEl - EntS00) traduit une appartenance collective qui va au delà des groupes nationaux qui renvoient aux seuls pays du Maghreb. Figure transnationale, elle met en avant une saillance ethnique, processus par lequel des traits de catégorisation sont sélectionnés, mis en mots et rendus disponibles à l'intérieur et à l'extérieur du groupe auquel ils font référence. Dans ce cas-ci, se dire arabe est une manière de faire allégeance à un groupe, et plus précisément à un groupe minorisé socialement et à le réinvestir à l'encontre de la domination sociale qui l'essentialise. La deuxième figure identitaire qui se dessine mobilise les origines de la famille et la nationalité du jeune :

4/N-00 : (...) je suis d'origine algérienne Français

5/Enq : c'est important pour toi de dire que tu es d'origine algérienne Français?

6/N-00 : oui ça fait partie de moi quoi, de ma carte d'identité $7 /$ Enq : oui

$8 / \mathrm{N}-00$ : ça dit d'où je suis $(E n t E l-E n t N O 0)$.

310/Y-00 : je suis né français (...), mais mes parents ils sont Arabes

311/Enq : oui comme tu es Français d'origine arabe, est-ce qu'il y a quelque chose qui te différencie d'un Français et qui te différencie d'un Arabe? est-ce que tu vois une différence? est-ce qu'il y a une différence entre toi et un Arabe?

\footnotetext{
44 Danièle Moore, op. cit., p. 23.

45 Cécile Sabatier, "Appartenances identitaires... ", op. cit.
} 
312/Y-00 : oui

313/Enq : quelle est cette différence?

314/Y-00 : ils sont nés dans le pays arabe, et si tu leur dis «t'es de quelle origine, t'es de quelle nationalité " ça va être bizarre parce que lui il va te dire 'nationalité algérienne, origine algérienne' alors que moi, si il me demande je dirais 'nationalité française et d'origine algérienne' $(E n t E l-E n t Y 00)$.

La mention des parents renvoie à l'importance de la symbolique langagière dans la construction des identités complexes, alors que la mention de la nationalité inscrit les jeunes dans une histoire et un parcours de vie différents de celui de leurs aînés. La troisième figure, que résume l'affirmation de Mo-01 - « Je suis Français-Tunisien " (296/Mo-01 - EntEl- Mo-01), prononcée dans une seule continuité que nous avons visualisée graphiquement par un signe typographique matérialisant la liaison entre éléments - met bien en évidence une construction identitaire ressentie et articulée autour d'appartenances multiples, mais aussi possibles, qui relient explicitement les différents univers sociaux et culturels auxquels les sujets disent appartenir, alternativement et/ou conjointement ${ }^{46}$. L'intégrité des appartenances souligne un équilibre entre la perception que le sujet a de lui-même et les rôles et statuts que les effets de catégorisation sociale lui attribuent. Ces appartenances entières ne sont ni rigides, ni données une fois pour toutes; elles se (re)composent selon une gestion pragmatique des différents éléments qui les façonnent et au gré des réseaux sociaux dans lesquels les jeunes évoluent. Enfin, elles sont constamment soumises aux interactions et à l'environnement sociolinguistique et culturel lui-même en mouvement.

En nous attachant aux représentations des jeunes sur les langues en présence dans leurs répertoires verbaux et en les mettant en synergie avec les affirmations identitaires déclarées, il est enfin apparu des tensions entre les individus et les langues en contact. Objets de communication verbale qu'il s'agit de maîtriser et de sauvegarder, marqueurs et vecteurs d'identités qui bousculent les

\footnotetext{
$\overline{46}$ Cécile Sabatier, « Figures identitaires... », op. cit.; Cécile Sabatier, « Appartenances identitaires..." " op. cit.
} 
modes d'appréhension habituelle du monde, les langues en contact au sein des répertoires verbaux des jeunes sont porteuses de valeurs et de statuts qui témoignent de la nécessité de comprendre les contextes du développement du plurilinguisme, mais également de réinterroger les éléments définitoires des concepts aujourd'hui en circulation pour caractériser le plurilinguisme des locuteurs.

\subsection{Des plurilingues qui s'ignorent}

En adoptant, en effet, un regard critique sur la façon dont les jeunes se sont positionnés tout au fil des entretiens à la fois au regard des thèmes et aussi des concepts sous-jacents discutés, il ressort que les conceptions des sujets et les définitions des sociolinguistes pour caractériser le plurilinguisme des locuteurs ne sont pas toujours similaires et que les représentations sociales dominantes sont très prégnantes, y compris dans les discours des participants, surtout lorsqu'il est question de langues qui renvoient à des communautés socialement minorisées ${ }^{47}$. Les représentations des jeunes sur leur plurilinguisme illustrent cet écart, car à la question de savoir s'ils se considéraient comme des individus plurilingues, seuls trois des onze sujets ont répondu par l'affirmatif. La majorité des jeunes, à l'instar de H-01 et de A-01, est hésitante quant à se dire bi-/plurilingue :

181/Enq : oui, alors toi est-ce que tu te considères comme bilingue?

182/H-01: ... je sais pas (EntEl-EntH01).

347/Enq : (...) alors, est ce que toi tu te considères comme bilingue, est-ce que tu es bilingue?

348/A-01 : ... bilingue, c'est qu'on utilise plusieurs langues?

349/Enq : oui deux

350/A-01: ... ben un petit peu oui

352/A-01 : ben parce que (...) c'est vrai quand même que je parle, c'est vrai que je parle français, mais arabe je connais pas tous les mots qui existent, alors voilà, c'est pour ça $(E n t E l-E n t A 01)$.

$47 \quad$ Christine Hélot, Du bilinguisme en famille au plurilinguisme à l'école, Paris, L'Harmattan, 2008. 
Entre confusion et insécurité linguistique d'un côté, acceptation de l'autre du discours social ambiant qui qualifie de bi/ plurilingues des individus dont les langues familiales sont fortement valorisées dans la société et qui ont une maîtrise des idiomes équivalente, et bien que témoignant de pratiques langagières plurilingues, les jeunes ne s'inscrivent pas ouvertement dans une approche des plurilinguismes en termes de gestion d'un capital linguistique (et communicatif) issu de plusieurs langues, en fonction des différentes situations de communication et de vie quotidienne auxquelles ils sont confrontés, et ceci, sans qu'il y ait un équilibre parfait entre les compétences. Au contraire, ils laissent transparaître, comme les propos de A-01 ci-dessous le montrent, une prégnance des discours qui soulignent davantage une définition du concept de bilinguisme en termes de maîtrise (si possible équilibrée) de deux systèmes linguistiques, plutôt qu'une approche qui met l'accent sur les répertoires pluriels, asymétriques, mais dynamiques des individus au contact de plusieurs langues :

352/A-01 : ben parce que (...) c'est vrai quand même que je parle, c'est vrai que je parle français, mais arabe je connais pas tous les mots qui existent, alors voilà, c'est pour ça $(E n t E l-E n t A 01)$

En effet, bien que vivant au quotidien le contact des langues sur le mode de la complémentarité, compte tenu des fonctions remplies par les divers idiomes et des réseaux sociaux dans lesquels ils sont activés, tout en ayant la capacité de mobiliser (ou d'occulter) l'ensemble des langues de manière homogène, les jeunes ne semblent pas avoir une représentation plurilingue de leurs pratiques langagières et par conséquent de leurs plurilinguismes. Le maintien d'éléments définitoires qui ont trait à la manière monolingue dont les premières études ${ }^{48}$ sur le bilinguisme caractérisaient ce dernier, souligne la difficulté pour ces jeunes, mais aussi pour la société environnante, d'appréhender le développement des plurilinguismes individuels dans des schèmes d'interprétation qui laissent la place au déséquilibre, à la recon-

$48 \quad$ Nous faisons ici allusion aux premiers travaux sur le bilinguisme de Ronjat, (1913), de Leopold (1930) et de Bloomfield (1933), entre autres. 
figuration systémique des langues en contact, aux circulations entre langues et cultures, à des combinaisons possibles de compétences et aux affirmations identitaires plurielles.

Mettre l'accent sur des répertoires pluriels, asymétriques, dynamiques, instables, c'est aussi émettre des doutes face à des conceptions 'unilingues' de la langue, du locuteur/auditeur idéal, de la communauté linguistique. Et si le locuteur/auditeur idéal vivait dans une société plurilingue hétérogène? (Lüdi, 2000 : 188).

\section{Conclusion}

$\mathrm{Au}$ fil des discours des adolescents, les pratiques langagières déclarées en familles, entre amis, au sein plus largement des différents réseaux sociaux dans lesquels ils sont insérés, font montre d'une multitude de possibilités entre les divers idiomes en contact au sein des répertoires verbaux. Ces choix sont directement corrélés avec les contextes de communication mais aussi les identités linguistiques, culturelles et sociales que les adolescents veulent affirmer. Toutefois, lorsqu'ils sont invités à se pencher sur leur vécu sociolangagier et à utiliser des mots de spécialité, les mots des linguistes, les adolescents se montrent réticents à employer de tels lexèmes. Ainsi, une contradiction entre leurs pratiques de facto plurilingues, et leurs représentations liées au plurilinguisme et au contact de langues, plus conformes à celles du discours social dominant apparaît clairement.

Ce décalage conduit les spécialistes en sciences sociales à s'interroger conséquemment sur les façons de caractériser les situations complexes de contact et sur les mots en circulation pour décrire mais aussi pour appréhender de telles situations. Le regard ainsi porté aux pratiques (socio)langagières déclarées et aux répertoires verbaux que des jeunes développent au contact de plusieurs langues, de plusieurs cultures et de plusieurs réseaux sociaux au quotidien ${ }^{49}$, nous conduit en conclusion à prôner un déplacement de paradigme dans la manière d'envisager la

49 Cécile Sabatier, «Compétence plurilingue et dynamiques d'appropriation langagière ", dans Danièle Moore et Véronique Castellotti (dir.), La compétence plurilingue. Regards francophones, Berne, Peter Lang, 2007, p. 105126. 
construction même des plurilinguismes individuels dans des contextes sociaux globaux de plus en plus marqués par la diversité linguistique et culturelle.

Ce déplacement du regard vers un paradigme plurilingue ${ }^{50}$ modifie conséquemment les ancrages épistémologiques dans lequel s'ancrent les études et les outils à disposition pour comprendre la construction des plurilinguismes individuels dans leurs dynamiques et récursivités. Il interroge également les processus de transformation sociale, en permettant de porter attention aux conditions globales et locales qui permettent aux interlocuteurs de mobiliser les ressources de leurs répertoires pluriels. Les dimensions explicatives qui facilitent l'interprétation des paramètres contextuels, à savoir les dimensions systémique, sociolinguistique, interactionniste et psycholinguistique, sont plus que jamais des composantes majeures pour saisir la complexité des pratiques langagières et des constructions identitaires plurielles.

Au regard du domaine de l'éducation dans lequel le pluralisme linguistique et culturel de la clientèle scolaire fait aujourd'hui partie de la réalité des salles de classes des sociétés contemporaines, la compréhension émique des nouvelles formes de rapports aux langues, aux cultures et aux identités conduit à repenser le rôle que joue l'école dans la façon dont les élèves - et en particulier ceux avec un capital d'expériences linguistiques et culturelles varié - se positionnent dans leurs rapports à ces dernières. Ce nouvel éclairage s'inscrit dès lors à son tour dans de nouvelles perspectives sociales quant aux manières (épistémiques et pragmatiques) dont des individus évoquent et éprouvent leurs appartenances sociales en contextes. Le défi reste de savoir comment passer de l'individu, de ce que nos recherches nous disent de lui, à la question de l'aménagement politique. Comment franchir le pas? Quelle nécessaire réflexion doit-on mener pour parvenir, par delà de la singularité des parcours individuels, à dégager certains traits saillants qui redéfinissent de nouvelles

50 Laurent Gajo, Immersion, bilinguisme et interaction en classe, Paris, Didier, 2001; Georges Lüdi, "Synthèse : construire des répertoires pluriels dans l'interaction ", Notions en Questions, 4, 2000, p. 179-190; Danièle Moore, op. cit.; Philip Herdina et Ulrike Jessner, op. cit. 
conceptions des contacts de langues, dans les liens étroits qu'ils tissent avec la complexité?

\section{Conventions de transcription}

$1 /$ = tour de parole

Enq = enquêtrice

Mo-01 = code de l'élève identifié par les initiales de son prénom suivies de l'année d'enregistrement des données

$\mathrm{NON}=$ insistance sur le mot

$\ldots$ = pause / silence

$\mathrm{xxx}=$ inaudible

[les langues opprimées $\mathrm{xxx}$ ] = énoncé incertain + inaudible

(rires) $=$ commentaires $/$ précisions de l'enquêtrice

beau- = mot inachevé

\section{Bibliographie}

Bialystok, Ellen, Bilingualism in Development. Language, Literacy and Cognition, Cambridge, Cambridge University Press, 2001.

Billiez, Jacqueline (coord.), Une semaine dans la vie plurilingue à Grenoble, Observatoire des pratiques linguistiques, DGLF, Grenoble, Lidilem (non publié), 2000.

Blanchet, Philippe, Danièle Moore et Safia Assalah Rahal (dir.), Perspectives pour une didactique des langues contextualisée, Paris, Éditions des Archives Contemporaines et AUF, 2008.

Bloomfield, Leonard, Languages, London, Allen and Unwin, 1933.

Boggards, Paul, Aptitude et affectivité dans l'apprentissage des langues étrangères, Paris, Crédif / Hatier, 1988.

Boutet, Josiane et Claire Saillard, «Dynamique des répertoires linguistiques dans la migration chinoise", dans Jacqueline Billiez (dir.), Contacts de langues. Modèles, typologies, interventions, Paris, L'Harmattan, 2003, p. 91-109.

Boyer, Henri, Langues en conflit. Études sociolinguistiques, Paris, L'Harmattan, 1991. 
Boyer, Henri, Plurilinguisme : "contact " ou "conflit " de langues? Paris, L'Harmattan, 1997.

Calvet, Louis-Jean, Pour une écologie des langues du monde, Paris, Plon, 1999.

Canac-Marquis, Réjean et Cécile Sabatier, "Compétence plurilingue en contexte formel d'immersion (FL2) et réflexion curriculaire sur la formation des enseignants de français : quand la recherche se met au service de la salle de classe ", Le Journal de l'Immersion / Immersion Journal, vol. 31, n 3, 2009.

Candelier, Michel (dir.), L'éveil aux langues à l'école primaire. Evlang : bilan d'une innovation européenne, Bruxelles, De Boeck, 2003.

Castellotti, Véronique, D'une langue à d'autres : pratiques et représentations, Rouen, Publications de l'Université de Rouen, 2001.

Dagenais, Diane, Françoise Armand, Erica Maraillet et Nathalie Walsh, "L'Éveil aux langues et la co-construction de connaissances sur la diversité linguistique, Revue canadienne de linguistique appliquée, vol. 10, no 2, 2007, p. 197-219.

Dagenais, Diane, Danièle Moore, Cécile Sabatier, Patricia Lamarre et Françoise Armand, "Linguistic Landscape and Language Awareness", dans Durk Gorter et Elana Shohamy (dir.), Linguistic Landscape: Expanding The Scenery, LEA Routledge, 2009, p. 253-269.

Deprez, Christine, Les enfants bilingues : langues et familles, Paris, Crédif Didier, 1994.

Deprez, Christine, " Pour une conception plus circulante des langues mises en jeu dans les migrations ", dans Louis-Jean Calvet et Auguste Moussirou-Mouyama (dir.), Le plurilinguisme urbain, Paris, Institut de la Francophonie, Didier Érudition, 2000, p. 55-67.

Fishman, Joshua A., "Who speaks what language to whom and when? " La linguistique, vol. 2, 1965, p. 67-88

Gajo, Laurent, Immersion, bilinguisme et interaction en classe, Paris, Didier, 2001.

Galligani, Stéphanie et Cécile Sabatier, "Si on dit Mélanie... ça fait Méfélafanifi - un exemple de parler urbain : la 'langue de /fØ/" ", LIDIL, 19, 1999, p. 43-57.

Grosjean, François, "The bilingual's language modes », dans Janet Nicol (dir.), One Mind Two Languages, Oxford, Blackwell, 2001, p. 1-22.

Gumperz, John, Engager la conversation. Introduction à la sociolinguistique interactionnelle, Paris, Minuit, 1989.

Hélot, Christine, Du bilinguisme en famille au plurilinguisme à l'école, Paris, L'Harmattan, 2008. 
Herdina, Philip et Ulrike Jessner, A Dynamic Model of Multilingualism Perspectives of Change in Psycholinguistics, Clevedon, Multilingual Matters, 2002.

Hoffmann, Charlotte et Jehannes Ytsma (dir.), Trilingualism in Family, School and Community, Clevedon, Multilingual Matters, 2004.

Hymes, Dell, Vers la compétence de communication, Paris, Crédif / Hatier, Collection LAL, 1984.

Jodelet, Denise (dir.), Les représentations sociales, Paris, Presses universitaires de France, 1989.

Kaufmann, Jean-Claude, L'invention de Soi. Une théorie de l'identité, Paris, Hachette, 2004.

Kaufmann, Jean-Claude, L'entretien compréhensif, Paris, Nathan Université, 1996.

Labov, William, Sociolinguistique, Paris, Minuit, 1976.

Labov, William, Le parler ordinaire, Paris Éditions de Minuit (édition originale, 1972, University of Pennsylvania Press), 1978.

Lamarre, Patricia et Diane Dagenais, "Trilingual youth in Canada ", dans Charlotte Hoffmann et Jehannes Ytsma (dir.), Trilingualism in Family, School and Community, Clevedon, Multilingual Matters, 2004, p. 53-74.

Leconte, Fabienne et Clara Mortamet, "La construction des identités plurilingues d'adolescents nouvellement arrivés en France ", dans Pierre Martinez, Danièle Moore et Valérie Spaëth (dir.), Plurilinguismes et enseignement. Identités en construction, Paris, Riveneuve Éditions, 2008, p. 167-177.

Leopold, Werner, Speech Development of a Bilingual Child. A Linguist's Record, Evanston, Northwestern University, 1939-1949.

Li, Wei, Three Generations Two Languages One Family: Language choice and language shift in a Chinese community in Britain, Clevedon, Multilingual Matters, 1994.

Lüdi, Georges, «Synthèse : construire des répertoires pluriels dans l'interaction », Notions en Questions, 4, 2000, p. 179-190.

Lüdi, Georges et Bernard Py, Etre bilingue, Berne, Peter Lang, 1986.

Lüdi, Georges et Bernard Py, Changement de langage et langage du changement. Aspects linguistiques de la migration interne en Suisse, Lausanne, L'Âge d'Homme, 1995.

Marshall, Steve, "Langues et identités dans les interactions entre les minorités allochtones et autochtones : essentialisme, appropriation et multiplicité en Catalogne ", dans Pierre Martinez, Danièle Moore et Valérie Spaëth (dir.), Plurilinguismes et enseignement. Identités en 
construction, Paris, Riveneuve Éditions, 2008, p. 77-88.

Martin-Jones, Marilyn, "Les modèles sociolinguistiques dans l'étude du bilinguisme : le cas des minorités linguistiques ", intervention dans le cadre du colloque, Contacts de Langues - Quels Modèles?, Nice, 28-30 septembre 1987, Paris, Maison des Sciences de l'Homme.

Milroy, Lesley, Language and Social Networks, $2^{\mathrm{e}}$ édition, Oxford, Blackwell, 1987.

Milroy, Lesley et Wei Li, «A social network approach to code-switching ", dans Lesley Milroy et Pieter Mysken (dir.), One Speaker Two Languages: Cross-disciplinary perspectives on code-switching, Cambridge, Cambridge University Press, 1995, p. 136-157.

Milroy, Lesley et Pieter Muysken (dir.), One Speaker Two Languages: Crossdisciplinary perspectives on code-switching, Cambridge, Cambridge University Press, 1995.

Moore, Danièle, Plurilinguismes et école, Paris, Didier, 2006.

Moore, Danièle et Claudine Brohy, "Identités plurilingues, dans Sociolinguistique des langues en contact, modèles, théories. Dictionnaire encyclopédique des termes et concepts, Lyon, Ens-Éditions (à paraître).

Morin, Edgar, Introduction à la pensée complexe, Paris, Éditions du Seuil, 2005.

Norton, Bonny, Identity and Language Learning: Gender, Ethnicity and Social Change, Harlow, Longman, 2000.

Pavlenko, Aneta et Adrian Blackledge, Negotiation of Identities in Multilingual Contexts, Clevedon, Multilingual Matters, 2004.

Poplack, Shana, "Sometimes I'll start a sentence in Spanish y termino en español ", Linguistics, 18, 1980, p. 581-618.

Py, Bernard, « Migration, apprentissage et réorganisation des compétences linguistiques ", dans Georges Lüdi et Bernard Py (dir.), Changement de langage et langage du changement. Aspects linguistiques de la migration interne en Suisse, Lausanne, L'Âge d'Homme, 1995, p. 117-146.

Rampton, Ben, Crossing: Language and Ethnicity among Adolescents, London, Longman, 1995.

Ronjat, Jules, Le développement du langage observé chez un enfant bilingue, Paris, Champion, 1913.

Sabatier, Cécile, «Appartenances identitaires de jeunes Français-Maghrébins à l'école élémentaire française. Entre maux et pratiques ", Child Heath Education / Santé et éducation de l'enfance, Revue en ligne, (à paraître).

Sabatier, Cécile, " "La puce à l'oreille". Représentations d'apprenants sur des sonorités langagières plus ou moins familières ", Revue de l'AQE- 
FLS, numéro spécial « Enseignement et acquisition de la phonétique en classe de langue seconde et étrangère ", 2009, vol. 27, n 1 , p. 112-133.

Sabatier, Cécile, "Compétence plurilingue et dynamiques d'appropriation langagière ", dans Danièle Moore et Véronique Castellotti (dir.), La compétence plurilingue. Regards francophones, Berne, Peter Lang, 2007, p. $105-126$.

Sabatier, Cécile, "Figures identitaires des élèves issus de la migration maghrébine à l'école élémentaire en France ", Éducation et Francophonie, vol. 34, no 1, 2006a, p. 111-132.

Sabatier, Cécile, "Symbolique des lieux et structuration linguistique de l'espace scolaire : comment les élèves redessinent les frontières de langues à l'école ", dans Christine. Hélot (dir.), Écarts de langues, écarts de cultures. À l'école de l'Autre, Berne, Peter Lang, 2006b, p. 75-86.

Sabatier, Cécile, Rôle de l'école dans le développement et la construction du plurilinguisme chez des enfants issus de la migration maghrébine en France, Lille, ANRT, 2004.

Schieffelin, Bambi et Elinor Ochs, "Language Socialization ", Annual Review of Anthropology, vol. 15, 1986, p. 163-191.

Weinreich, Uriel, «Unilinguisme et multilinguisme », dans André Martinet (dir.), Le Langage. Encyclopédie de la Pléiade, Paris, Gallimard, 1968. 